\title{
Antineoplastic Actions of Cinnamic Acids and Their Dimers in Breast Cancer Cells: A Comparative Study
}

\author{
MEGAN HUNKE ${ }^{1}$, WUILIAN MARTINEZ ${ }^{2}$, AKSHAY KASHYAP ${ }^{2}$, TREYVON BOKOSKIE ${ }^{2}$, \\ MAHESH PATTABIRAMAN ${ }^{2}$ and SURABHI CHANDRA ${ }^{1}$ \\ ${ }^{1}$ Department of Biology, University of Nebraska-Kearney, Kearney, NE, U.S.A.; \\ ${ }^{2}$ Department of Chemistry, University of Nebraska-Kearney, Kearney, NE, U.S.A.
}

\begin{abstract}
Background/Aim: Breast cancer is the second leading cause of cancer-related deaths in US, which necessitates constant research for medications with minimal adverse effects. The aim of the study was to determine if certain cinnamic acid dimers (CADs) exhibit higher cytotoxicity in breast cancer cells than against nontumorigenic cells. Materials and Methods: These dimers have not been explored, so far, due to their complex stereochemistry; cavitand-mediated photodimerization (CMP) method was used to produce several CADs, which were tested for cytotoxicity, apoptosis and cell cycle inhibition. Results: CADs such as ferulic acid dimer, 3-fluoro $C A D$, and 3, 4-difluoro $C A D$, were found to be more cytotoxic than their parent monomers. The most potent compound, 3-fluoro CAD caused apoptosis and 'S' phase cell cycle arrest in cancer cells. Conclusion: This study indicates an avenue for research for developing a novel class of antineoplastic compounds that can be synthesized using an easy, economical method.
\end{abstract}

In the United States, over 1.7 million new cancer cases are expected to be diagnosed in 2018, and around 610,000 Americans are estimated to die of cancer in 2018 (1). Breast cancer is of specific concern, as it is the most commonly diagnosed cancer in women and is the second leading cause of cancer deaths (2). The trend for breast cancer-related mortality has experienced a sharp decline of 39\% from 1989 to 2015 due to early detection and treatment (1). Nevertheless, increased life expectancies, irregular or prolonged menstrual history, genetic predisposition, in

Correspondence to: Surabhi Chandra, Ph.D., BHS335, Department of Biology, University of Nebraska-Kearney, 2401 W. 11th Ave, Kearney, NE 68849, U.S.A. Tel: +1 3088658661, Fax: +1 3088658045, e-mail: chandras2@unk.edu

Key Words: Cinnamic acid dimer, breast cancer cells, photodimerization, apoptosis, cell cycle arrest. combination with current lifestyles involving use of hormone therapies, excessive alcohol consumption, stress, etc. have ensued higher incidences of breast cancer (1). Efficient treatment options need to be investigated to minimize burden on the patient in their path to recovery.

Natural products have been widely researched for their anticancer properties, primarily as chemopreventive and chemotherapeutic agents. Synthetic derivatives of the pharmacoactive moiety of these natural products have also been investigated. Cinnamic acids (CA) are aromatic fatty acid phenylalanine deamination products naturally found in cinnamon, coffee beans, olives, fruits, and vegetables (3). CAs and their derivatives such as ferulic acid, caffeic acid, sinapic acid, chlorogenic acid, and isoferulic acid have been shown to possess antioxidant (4), anti-inflammatory (5), and anti-tumor (1) properties. The cis- and trans-isoforms of CAs have been evaluated regarding their effectiveness in inhibiting invasive lung adenocarcinoma cells (6). Underlying mechanisms for the cytotoxic action of CAs include inhibition of DNA synthesis, suppression of NF-kB activation, production of IL-8, inhibition of histone deacetylase, and antioxidant effects (3).

CAs are modified by gut microbiota to a biologically more active compound (7). Large variations in the cytotoxicity of CA derivatives is primarily due to chemical modifications on the parent molecule. It has been shown that the catechol ring and the hydroxylic group are associated with toxicity, but the effect is further enhanced by substitution of higher number of hydroxylic groups or alkyl chains or esterification of CA $(3,7-9)$.

Though research on plant-based phenolic compounds and their derivatives has received great attention for chemotherapeutics, so far there are no published reports on synthesis and biological actions of dimeric CAs. A recent report indicated that naturally-occurring eucommicin $\mathrm{A}$ (isolated from a Chinese plant), which is a diester of tetrahydroxy- $\beta$-truxinic acid (CA dimer), inhibits the growth of cancer stem cells (10). Here we report the cytotoxicity of CADs, synthesized using cavitand-mediated-approach in 


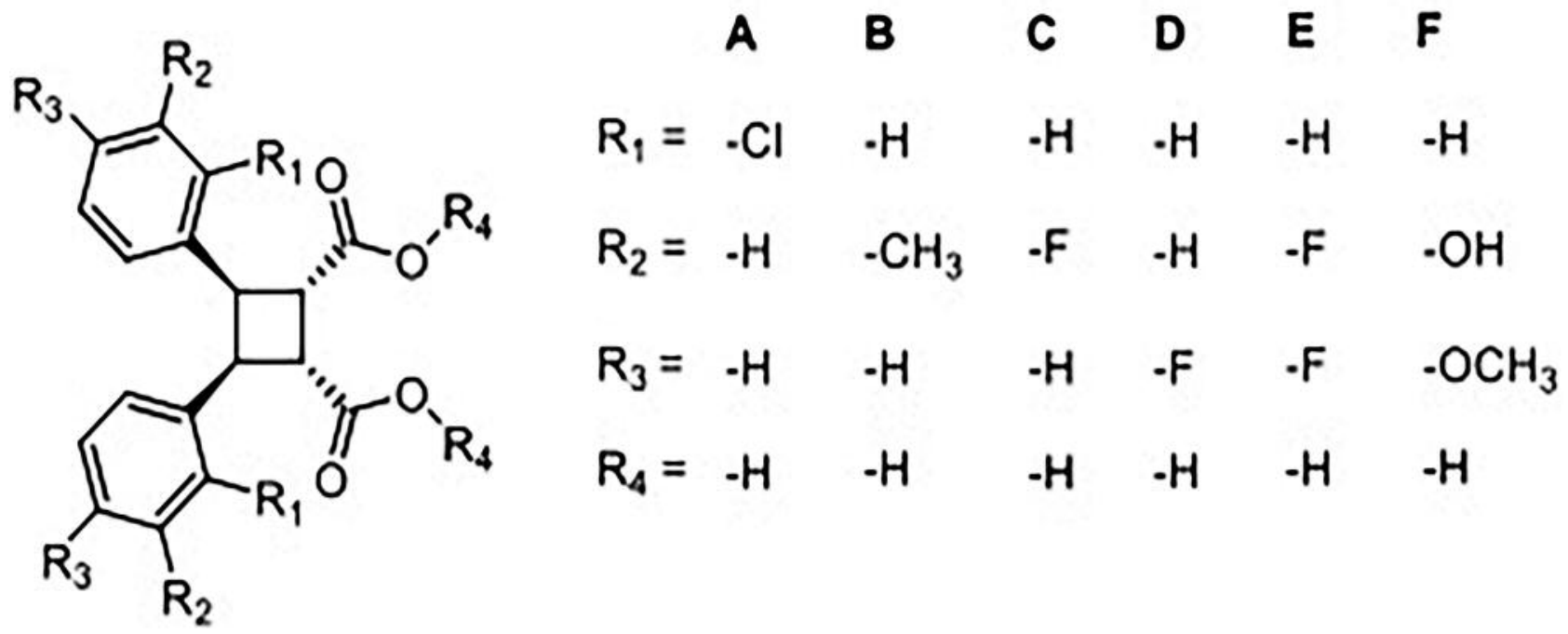

Figure 1. Chemical structures of CAD-Ds synthesized by CMP method: (A) 2-chloro CA (B) 3-methyl CA (C) 3-fluoro CA, (D) 4-fluoro CA, (E) 3,4-difluoro CA, (F) Ferulic Acid.

breast cancer cells. Our hypothesis is that CADs provide a unique class of potent chemotherapeutics against breast cancer cells, that can be synthesized using an efficient and economical synthetic procedure.

\section{Materials and Methods}

Chemicals and synthesis of dimers. Media supplements and all chemicals for dimer synthesis were obtained from Millipore Sigma (Milwaukee, WI, USA) or Peprotech (Rocky Hill, NJ, USA). DMEM/F12 media, horse serum, penicillin/streptomycin and GlutaMAX were obtained from Thermo Fisher Scientific (Rockford, IL, USA). Fetal bovine serum (FBS) was obtained from ATCC (Manassas, VA, USA). CADs were synthesized using cavitandmediated-photodimerization method (CMP) as reported earlier (11).

Cell culture and treatment. Breast cancer cells- MCF-7, MDA-MB231, and normal mammary epithelial cells, MCF-10A were purchased from ATCC, and cultured at $37^{\circ} \mathrm{C}$ in a humidified atmosphere with $5 \% \quad \mathrm{CO}_{2}$. MCF-10A cells were grown in DMEM/F12 media supplemented with $5 \%$ horse serum, $20 \mathrm{ng} / \mathrm{ml}$ epidermal growth factor, $0.5 \mu \mathrm{g} / \mathrm{ml}$ hydrocortisone, $100 \mathrm{ng} / \mathrm{ml}$ cholera toxin, $10 \mu \mathrm{g} / \mathrm{ml}$ insulin, $100 \mathrm{IU} / \mathrm{ml}$ penicillin, and $100 \mu \mathrm{g} / \mathrm{ml}$ streptomycin. MCF-7 cells and MDA-MB-231 cells were grown in DMEM/F12 supplemented with 5\% fetal bovine serum (FBS), 100 $\mathrm{IU} / \mathrm{ml}$ penicillin, and $100 \mu \mathrm{g} / \mathrm{ml}$ streptomycin. Treatment (24-72 h) with test compounds was performed using media lacking phenol red, as it interferes with dyes used for analyses.

Cell survival assay. Cells were grown in 96-well plates and treated with different concentrations of CADs and their parent monomers for 24-72 h. Vehicle control wells were treated with equivalent concentrations of acetone used for preparing stock solutions. Cell survival was measured using PrestoBlue dye (Thermo Fisher
Scientific, Rockford, IL, USA) as per manufacturer's instructions, and fluorescence was measured at 560/590 nm (ex/em) using a microplate reader and Gen5 software (BioTek Synergy 2; Winooski, VT, USA). Percent change was calculated relative to the average for vehicle control treated cells.

Colony-forming clonogenic assay. Cells were grown in 6-well plates (2000 cells/well) and treated with test compounds for $72 \mathrm{~h}$ (time chosen for maximum toxicity). Following treatment period, wells were incubated with media without the test compound. Existing cells were allowed to establish colonies for 7 days. After this, the wells were washed, fixed with methanol for $15 \mathrm{~min}$, and stained with $0.1 \%$ crystal violet for $30 \mathrm{~min}$. Stain was removed by immersing and rinsing the plate in tap water, and letting the plates dry overnight. Colonies with 50 or more cells were counted using a stereomicroscope. Survival fraction was calculated for each well using the formula: Number of colonies formed/Number of cells plated.

Apoptosis assay and cell cycle analysis. Cells were grown and treated in chambered slides and apoptosis assay was performed using Alexa Fluor ${ }^{\circledR} 488$ Annexin V/Dead Cell Apoptosis Kit (Thermo Fisher Scientific) as per manufacturer's instructions. Fluorescent images were taken on confocal laser scanning microscope (Olympus FV3000) and analyzed using NIH ImageJ software. For cell cycle analysis, cells were stained with FxCycle ${ }^{\mathrm{TM}}$ PI/RNase staining solution (Thermo Fisher Scientific) and analyzed using Sony SH800Z Cell Sorter. Percentage of cells in each growth phase was determined using the SH800Z Sony software.

Statistical analysis. Statistical analysis was performed using Graph Pad software (Prism 5.0). All experiments were performed at least three times in replicates. Comparison between two or more groups was performed using one-way ANOVA followed by Tukey's test. Differences were considered to be statistically significant at $p<0.05$. 


\section{Results}

Synthesis of CADs. CMP method using cavitands, such as $\gamma$ cyclodextrin $(\gamma$-CD) resulted in the formation of a single dimer, syn $\mathrm{H}-\mathrm{H}$ in high yields $(\sim 70 \%)$. CADs used in this study are listed in Figure 1. The choice of compounds was based on anticancer properties of parent CA monomers: ferulic acid, 2-chloro CA, and 3-methyl CA (12). Fluoro CAs were chosen as they have not been investigated for anticancer effects to our knowledge.

Effect of CAMs and CADs on survival of MCF-7, MDA-MB231, and MCF-10A cells. Survival curves in the presence of test compounds were generated for all cell lines upon treatment with CA monomers and CADs. In general, CADs were found to be more effective than their parent monomers, as evidenced by $\mathrm{IC}_{50}$ values in MCF-7 and MDA-MB-231 (Table I). Some CADs (ferulic acid, 3F CAD, and 3, 4-diF CAD) that had their $\mathrm{IC}_{50}$ values in $\mu \mathrm{M}$ (Tables I, II and III), were explored further. These compounds had at least three-five-fold higher $\mathrm{IC}_{50}$ values when tested in the non-tumorigenic epithelial cell line, MCF-10A. Dose-dependent cell survival curves generated for 3F CAD, 4F CAD, or 3, 4-diF CAD at different time points (24-72 h) (Figure 2) indicated that longer incubation (72 h) caused maximum inhibition of cell growth.

Effect of CADs on colony formation in MCF-7 and MDA-MB231 cells. Colony formation using clonogenic assay was used to assess in vitro tumor forming potential of $\mathrm{MCF}-7$ and MDA-MB231 cells in the presence of $3 \mathrm{~F} \mathrm{CAD}$ after $72 \mathrm{~h}$ exposure. Compared to vehicle control a concentration dependent inhibitory response in colony formation was observed (Figure 3).

Apoptosis assay and cell cycle analysis. Cancer cell lines were treated with $3 \mathrm{~F}$ CAD for $72 \mathrm{~h}$, and representative images of MDA-MB-231 cells are provided in Figure 4. The panel on the right is a merged image of apoptotic (green fluorescence, left panel) and necrotic cells (red fluorescence, middle panel). It was observed that while control cells did not exhibit significant apoptosis or necrosis, $25 \mu \mathrm{M}$ concentration resulted in higher levels of apoptosis, and $50 \mu \mathrm{M}$ concentration increased both apoptosis and necrosis.

Cell-cycle analysis indicated a proliferative difference between cancer cells and non-tumorigenic cells, as denoted by the higher $\%$ of treated cells in the ' $S$ ' phase (Table IV). Further, 3F CAD caused ' $S$ ' phase cell cycle arrest, with increasing number of cells in $\mathrm{S}$ phase, suggesting inhibition of DNA replication.

\section{Discussion}

Cinnamic acid has long been investigated for its biological properties including anti-inflammatory, antioxidant, and
Table I. $I C_{50}$ values (in $\mathrm{mM}$ ) in MCF-7 cells. Hyphens represent values $>5 \mathrm{mM}$.

\begin{tabular}{lccccccc}
\hline & \multicolumn{3}{c}{ Monomers } & & \multicolumn{3}{c}{ Dimers } \\
\cline { 2 - 4 } \cline { 7 - 8 } Test Compounds & $24 \mathrm{~h}$ & $48 \mathrm{~h}$ & $72 \mathrm{~h}$ & & $24 \mathrm{~h}$ & $48 \mathrm{~h}$ & $72 \mathrm{~h}$ \\
\hline 2-chloro CA & - & - & 3.2 & & 2.2 & 2.1 & 0.9 \\
3-methyl CA & - & 2.1 & 2.4 & & - & 1.2 & 1.1 \\
Ferulic Acid & 3.2 & 2.8 & 2.3 & & 0.6 & 0.5 & 0.5 \\
3-fluoro CA & - & - & - & & 1.6 & 0.3 & 0.1 \\
4-fluoro CA & - & - & - & & 2.9 & 0.9 & 0.9 \\
3,4-difluoro CA & - & 1.2 & 1.6 & & - & 0.9 & 0.8 \\
\hline
\end{tabular}

Table II. $I C_{50}$ values (in $\mathrm{mM}$ ) in MDA-MB-231 cells. Hyphens represent values $>5 \mathrm{mM}$.

\begin{tabular}{lcccccccc}
\hline & \multicolumn{3}{c}{ Monomers } & & \multicolumn{3}{c}{ Dimers } \\
\cline { 2 - 4 } \cline { 7 - 9 } Test Compounds & $24 \mathrm{~h}$ & $48 \mathrm{~h}$ & $72 \mathrm{~h}$ & & $24 \mathrm{~h}$ & $48 \mathrm{~h}$ & $72 \mathrm{~h}$ \\
\hline 2-chloro CA & - & 2.4 & 3.3 & & 0.7 & 0.7 & 0.6 \\
3-methyl CA & - & - & - & & 2.2 & 1.0 & 0.9 \\
Ferulic Acid & - & 3.3 & 2.3 & & 0.7 & 0.7 & 0.6 \\
3-fluoro CA & - & - & - & & 1.1 & 0.4 & 0.2 \\
4-fluoro CA & - & - & - & & 1.9 & 1.2 & 1.3 \\
3,4-difluoro CA & - & - & - & & 0.7 & 0.8 & 0.4 \\
\hline
\end{tabular}

Table III. $I C_{50}$ values (in $\mathrm{mM}$ ) in MCF-10A cells. Hyphens represent values $>5 \mathrm{mM}$.

\begin{tabular}{lcccccccc}
\hline & \multicolumn{3}{c}{ Monomers } & & \multicolumn{3}{c}{ Dimers } \\
\cline { 2 - 4 } \cline { 7 - 9 } Test Compounds & $24 \mathrm{~h}$ & $48 \mathrm{~h}$ & $72 \mathrm{~h}$ & & $24 \mathrm{~h}$ & $48 \mathrm{~h}$ & $72 \mathrm{~h}$ \\
\hline 2-chloro CA & - & 1.6 & 3.5 & & 2.0 & 1.9 & 1.3 \\
3-methyl CA & - & 2.4 & 2.4 & & 2.2 & 1.2 & 0.9 \\
Ferulic Acid & 3.8 & 2.9 & 1.9 & & 2.9 & 1.9 & 1.1 \\
3-fluoro CA & - & - & - & & 1.1 & 0.4 & 0.4 \\
4-fluoro CA & - & - & - & & 1.2 & 1.2 & 0.8 \\
3,4-difluoro CA & - & - & - & & - & 1.1 & 0.9 \\
\hline
\end{tabular}

anticancer effects $(5,6,13)$. Since parent compounds of CA and its derivatives exhibit low potency, research has been aimed towards synthesizing analogs with substitutions that increase lipophilicity and potentiate its action. A recent report studied the cytotoxicity of $\mathrm{CA}$ phenetyl esters and indicated that the potency against squamous cancer cell line was dependent on ionization potential, shape, and size of the CA (14). To our knowledge, we are the first to report the synthesis and chemotherapeutic actions of dimers of CA, which are effective even at half molar concentrations 
A



B

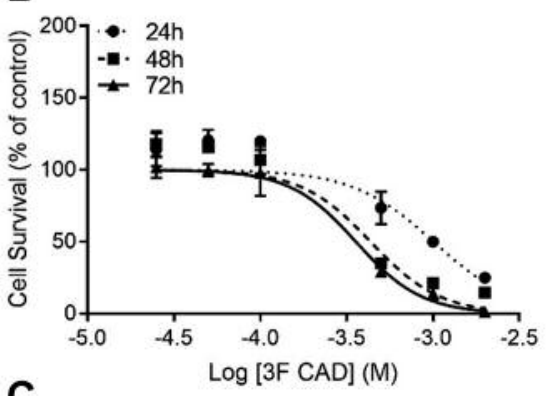

C

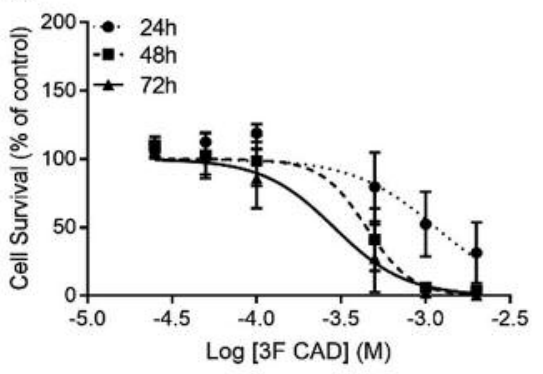

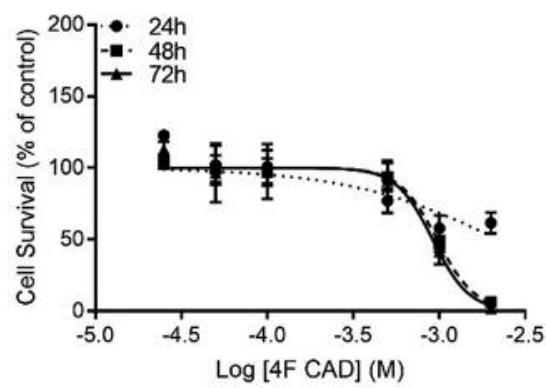
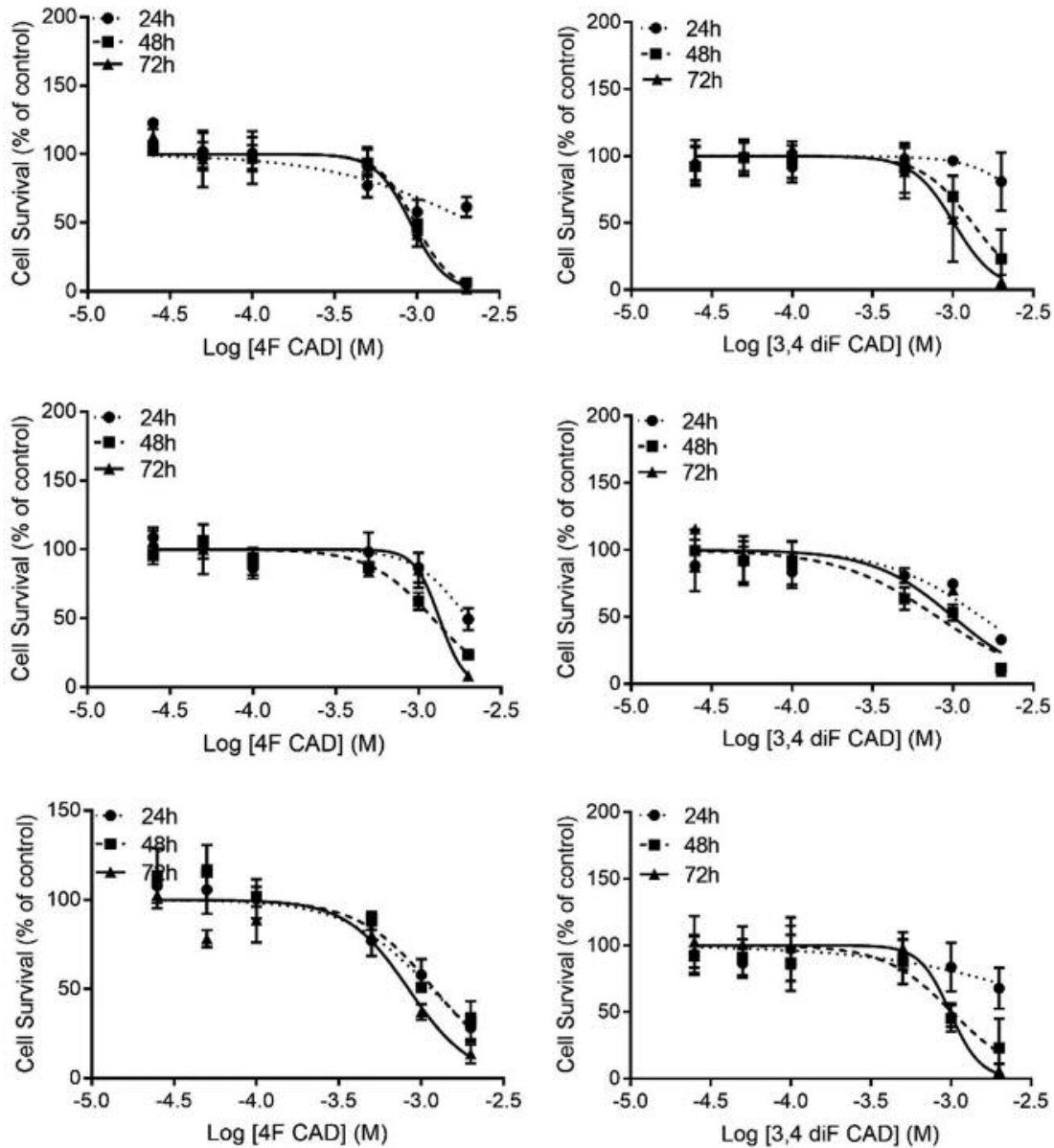

Figure 2. Survival curves of (A) MCF-7, (B) MDA-MB-231, and (C) MCF-10A cells treated with 3-fluoro CAD (3F CAD), 4-fluoro CAD (4F CAD), and 3, 4-difluoro CAD (3,4 diFCAD). Values are represented as average \pm SEM of triplicate determinations.

compared to their monomeric counterparts, despite same molecular mass. Only one recent study so far has reported that a natural syn H-H dimer of CA ( $\beta$-truxinic acid), eucommicin A, extracted from Eucommia ulmoides, exhibits anticancer property (10). They also suggested that the cyclobutane ring is essential for producing these effects. There has been no research so far on synthetic CADs for their anticancer actions. In addition, fluoro-substituted CAs have not been tested as yet for their cytotoxic actions in cancer cells.

The CMP method, used to synthesize the dimers used in our study, confines CA in the cavitand cavity in a specific orientation, thereby increasing photodimerization efficiency. Photodimerization yields four types of dimers: $s y n \mathrm{H}-\mathrm{H}$, syn $\mathrm{H}-\mathrm{T}$, anti $\mathrm{H}-\mathrm{H}$, and anti $\mathrm{H}-\mathrm{T}$ as reported earlier by our group (15-17). We could predominantly obtain the syn $\mathrm{H}-\mathrm{H}$ dimer using CMP method. A head-to-head $(\mathrm{H}-\mathrm{H})$ orientation is favored due to the stabilizing/attractive hydrogen-bonding (between the carboxylic acids of two CAs) and $\pi-\pi$ (between phenyl rings of two CAs) interactions.

Amongst the CADs tested, ferulic acid, 3-fluoro CAD, and 3,4-difluoro CAD were found to be the most cytotoxic to breast cancer cells, with little to no toxicity in nontumorigenic mammary epithelial cells at the same concentrations. CA monomer and its hydrophilic derivatives exhibit toxicity in the $\mathrm{mM}$ range $(3,12,13,18)$, however, most of the dimers tested here had their $\mathrm{IC}_{50}$ in the $\mu \mathrm{M}$ range. Due to the potent action of $3 \mathrm{~F}$ CAD compared to other compounds tested in the group, it was further explored for inhibition of colony formation, induction of apoptosis, and arrest of cell cycle. Interestingly, 3F CAD suppressed colony formation at a much lower dose than its $\mathrm{IC}_{50}$, as determined earlier by cell survival assay. This is possibly due to the smaller number of cells plated for colony formation, which could enhance toxicity with the compound. Percentage of apoptotic cells also increased at higher concentrations of 


\begin{tabular}{|c|c|c|c|}
\hline $3 \mathrm{~F} \mathrm{CAD}$ in $\mu \mathrm{M}$ & 0 & 25 & 50 \\
\hline \multicolumn{4}{|l|}{ MCF-7 Cells } \\
\hline Survival Fraction & $0.08 \pm 0.01$ & $0.05 \pm 0.01$ & $0.02 \pm 0.01$ \\
\hline \multicolumn{4}{|c|}{ MDA-MB-231 Cells } \\
\hline Survival Fraction & $0.09 \pm 0.02$ & $0.07 \pm 0.01$ & $0.04 \pm 0.02$ \\
\hline
\end{tabular}

Figure 3. Colony formation following treatment with $3 F C A D$ at $72 \mathrm{~h}$. Survival fraction values are represented as average $\pm S D$ of three replicates.

A

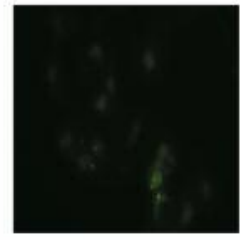

B

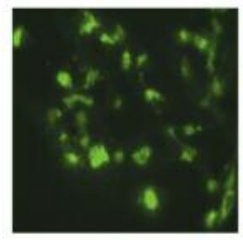

C
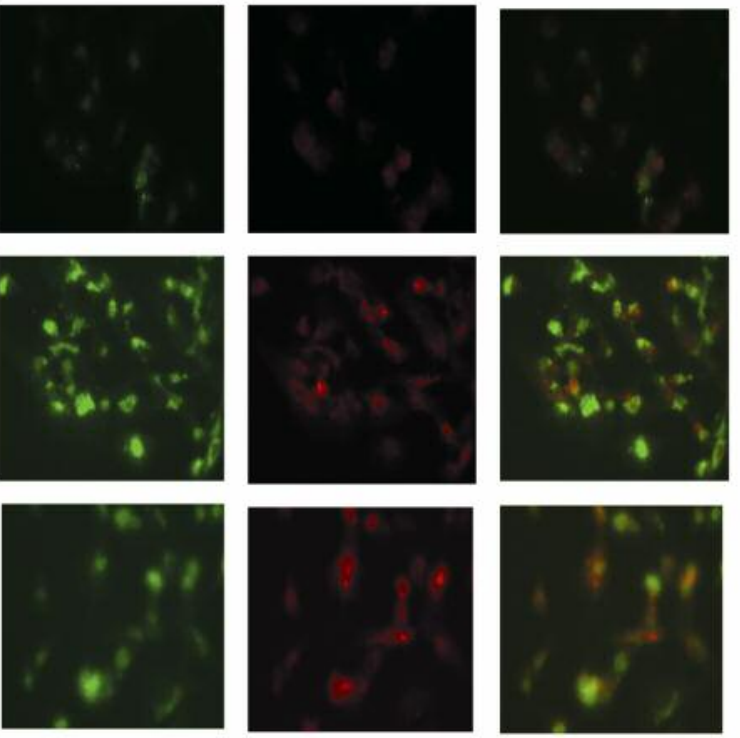

Figure 4. Apoptosis of MDA-MB-231 cells after $72 \mathrm{~h}$ treatment with $3 F$ $C A D$ at (A) $0 \mu M$, (B) $25 \mu M$, and (C) $50 \mu M$ concentrations. Left panel is green fluorescence representing apoptosis, middle panel is red fluorescence representing necrosis and right panel is merged image representing apoptotic and necrotic cells.

3F CAD in both cancer cell types. Representative image with MDA-MB-231 cells is provided. A study found that caffeic acid and ferulic acid (derivatives of CA) inhibit invasion, migration, and adhesion of lung adenocarcinoma cells (19). Another study concluded that propolis CA derivatives induced both extrinsic and intrinsic apoptosis signaling
Table IV. Distribution of cells in different phases of the cell cycle upon treatment with $3 F C A D(72 \mathrm{~h})$.

\begin{tabular}{lcccc}
\hline & & \multicolumn{3}{c}{$\%$ of cells in each phase } \\
\cline { 3 - 5 } Cell Type & 3F CAD $(\mu \mathrm{M})$ & $\mathrm{G}_{0} / \mathrm{G}_{1}$ & $\mathrm{~S}$ & $\mathrm{G}_{2} / \mathrm{M}$ \\
\hline \multirow{2}{*}{ MCF-7 } & 0 & 69.1 & 3.6 & 14.3 \\
& 25 & 68.1 & 5.1 & 14.5 \\
MDA-MB-231 & 50 & 67.4 & 5.3 & 15.2 \\
& 0 & 66.2 & 4.8 & 20.4 \\
MCF-10A & 25 & 68.6 & 6.1 & 19.3 \\
& 50 & 65.4 & 8.0 & 18.6 \\
& 0 & 91.5 & 1.1 & 7.4 \\
& 25 & 86.1 & 0.9 & 8.1 \\
& 50 & 88.3 & 1.3 & 7.6 \\
\hline
\end{tabular}

pathways against human colon cancer cells $(20,21)$. In human melanoma cells, CA induces apoptotic cell death by disrupting the cytoskeleton (18). Future studies will involve further investigation of the apoptotic pathway.

Cell cycle analysis using flow cytometry revealed that cancer cells have a higher percentage of cells in the replicative 'S' phase compared to MCF-10A non-tumorigenic cells. Furthermore, 3F CAD treatment significantly increased the number of MCF-7 and MDA-MB-231 cells in ' $S$ ' phase, which suggests $\mathrm{S}$ phase cell cycle arrest. Parent $\mathrm{CA}$ monomer has instead been shown to increase the $G_{0} / G_{1}$ phase and extend the cell cycle in leukemia, K562, HeLa, Fem-x, and MCF-7 cells (22). Future studies will also explore other substitutions in addition to the fluoro 
compounds examined in this study. The results of our study, as well as previously published research, warrant the potential use of CADs as cancer therapeutics. Our recent publication has shown that CADs also possess antinociceptive properties, which could help decrease pain felt by those undergoing cancer treatment (11). It is imperative to move forward and identify safe compound concentrations for use in vivo against cancer.

\section{Acknowledgements}

This study was funded by American Chemical Society Petroleum Research Fund (\# 54862-UR4) awarded to MP for the chemical synthesis. Biological studies were supported by the Great Plains IDeA CTR Pilot Grant (NIGMS \#1U54GM115458-01) awarded to MP and SC.

\section{References}

1 Bezerra DP, Castro FO, Alves AP, Pessoa C, Moraes MO, Silveira ER, Lima MA, Elmiro FJ and Costa-Lotufo LV: In vivo growth-inhibition of Sarcoma 180 by piplartine and piperine, two alkaloid amides from Piper. Braz J Med Biol Res 39(6): 801-807, 2006.

2 National Breast Cancer Foundation I. Breast Cancer Facts. 2016.

3 Su P, Shi Y, Wang J, Shen X and Zhang J: Anticancer agents derived from natural cinnamic acids. Anticancer Agents Med Chem 15(8): 980-987, 2015.

4 Chung HS and Shin JC: Characterization of antioxidant alkaloids and phenolic acids from anthocyanin-pigmented rice (Oryza sativa cv. Heugjinjubyeo). Food Chem 104(4): 1670-1677, 2007.

5 Kim EO, Min KJ, Kwon TK, Um BH, Moreau RA and Choi SW: Anti-inflammatory activity of hydroxycinnamic acid derivatives isolated from corn bran in lipopolysaccharidestimulated Raw 264.7 macrophages. Food Chem Toxicol 50(5): 1309-1316, 2012.

6 Yen GC, Chen YL, Sun FM, Chiang YL, Lu SH and Weng CJ: A comparative study on the effectiveness of cis- and trans-form of cinnamic acid treatments for inhibiting invasive activity of human lung adenocarcinoma cells. Eur J Pharm Sci 44(3): 281287, 2011.

7 Anantharaju PG, Gowda PC, Vimalambike MG and Madhunapantula SV: An overview on the role of dietary phenolics for the treatment of cancers. Nutr J 15(1): 99, 2016.

8 Serafim TL, Carvalho FS, Marques MP, Calheiros R, Silva T, Garrido J, Milhazes N, Borges F, Roleira F, Silva ET, Holy J and Oliveira PJ: Lipophilic caffeic and ferulic acid derivatives presenting cytotoxicity against human breast cancer cells. Chem Res Toxicol 24(5): 763-774, 2011.

9 Lee YJ, Liao PH, Chen WK and Yang CY: Preferential cytotoxicity of caffeic acid phenethyl ester analogues on oral cancer cells. Cancer Lett 153(1-2): 51-56, 2000.

10 Fujiwara A, Nishi M, Yoshida S, Hasegawa M, Yasuma C, Ryo A and Suzuki Y: Eucommicin A, a beta-truxinate lignan from Eucommia ulmoides, is a selective inhibitor of cancer stem cells. Phytochemistry 122: 139-145, 2016.
11 Priebe A, Hunke M, Tonello R, Sonawane Y, Berta T, Natarajan A, Bhuvanesh N, Pattabiraman M and Chandra S: Ferulic acid dimer as a non-opioid therapeutic for acute pain. J Pain Res 11: 1075-1085, 2018.

12 De P, Baltas M and Bedos-Belval F: Cinnamic acid derivatives as anticancer agents-a review. Curr Med Chem 18(11): 16721703, 2011.

13 Pontiki E, Hadjipavlou-Litina D, Litinas K and Geromichalos G: Novel cinnamic acid derivatives as antioxidant and anticancer agents: design, synthesis and modeling studies. Molecules 19(7): 9655-9674, 2014.

14 Uesawa Y, Sakagami H, Okudaira N, Toda K, Takao K, Kagaya $\mathrm{H}$ and Sugita Y: Quantitative structure-cytotoxicity relationship of cinnamic acid phenetyl esters. Anticancer Res 38(2): 817-823, 2018.

15 Nguyen N, Clements A and Pattabiraman M: Using non-covalent interactions to direct regioselective $2+2$ photocycloaddition within a macrocyclic cavitand. New J Chem 40(3): 2433-2443, 2016.

16 Pattabiraman M, Kaanumalle LS, Natarajan A and Ramamurthy V: Regioselective Photodimerization of Cinnamic Acids in Water: Templation with Cucurbiturils. Langmuir 22(18): 76057609, 2006.

17 Pattabiraman M, Natarajan A, Kaanumalle LS and Ramamurthy $\mathrm{V}$ : Templating photodimerization of trans-cinnamic acids with cucurbit[8]uril and gamma-cyclodextrin. Org Lett 7(4): 529-532, 2005.

18 Niero EL and Machado-Santelli GM: Cinnamic acid induces apoptotic cell death and cytoskeleton disruption in human melanoma cells. J Exp Clin Cancer Res 32: 31, 2013.

19 Tsai CM, Yen GC, Sun FM, Yang SF and Weng CJ: Assessment of the anti-invasion potential and mechanism of select cinnamic acid derivatives on human lung adenocarcinoma cells. Mol Pharm 10(5): 1890-1900, 2013.

20 Akao Y, Maruyama H, Matsumoto K, Ohguchi K, Nishizawa K, Sakamoto T, Araki Y, Mishima S and Nozawa Y: Cell growth inhibitory effect of cinnamic acid derivatives from propolis on human tumor cell lines. Biol Pharm Bull 26(7): 1057-1059, 2003.

21 Kumazaki M, Shinohara H, Taniguchi K, Yamada N, Ohta S, Ichihara $\mathrm{K}$ and Akao $\mathrm{Y}$ : Propolis cinnamic acid derivatives induce apoptosis through both extrinsic and intrinsic apoptosis signaling pathways and modulate of miRNA expression. Phytomedicine 21(8-9): 1070-7107, 2014.

22 Sova M, Zizak Z, Stankovic JA, Prijatelj M, Turk S, Juranic ZD, Mlinarič-Raščan I and Gobec S: Cinnamic acid derivatives induce cell cycle arrest in carcinoma cell lines. Med Chem 9(5): 633-641, 2013. 